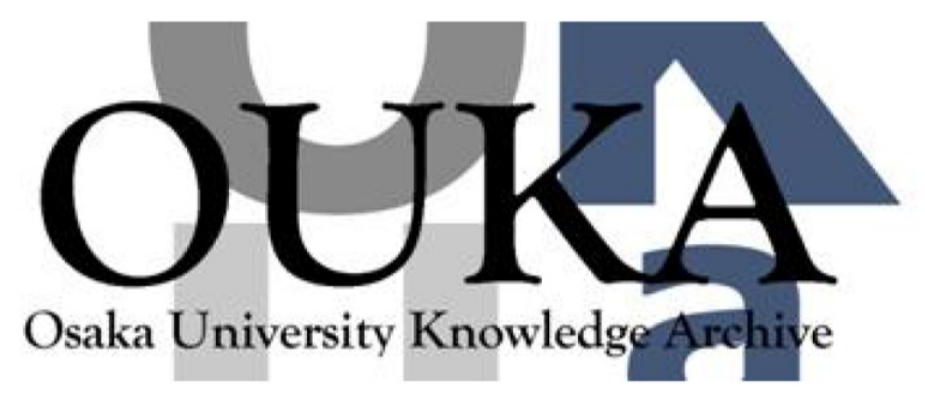

\begin{tabular}{|c|c|}
\hline Title & $\begin{array}{l}\text { Transport and Thermal Properties of Heavy- } \\
\text { Fermion Superconductors: A Unified Picture }\end{array}$ \\
\hline Author (s) & Schmitt-Rink, S.; Miyake, K.; Varma, C. M. \\
\hline Citation & Physical Review Letters. 57(20) p. 2575-p. 2578 \\
\hline Issue Date & $1986-11-17$ \\
\hline oaire:version & VoR \\
\hline URL & https://hdl. handle. net/11094/3012 \\
\hline rights & $\begin{array}{l}\text { Schmitt-Rink, S., Miyake, K., Varma, C. M., } \\
\text { Phys ical Review Letters, 57, 20, 2575-2578, } \\
\text { 1986-11-17. "Copyright 1986 by the American } \\
\text { Physical Society." }\end{array}$ \\
\hline Note & \\
\hline
\end{tabular}

Osaka University Knowledge Archive : OUKA

https://ir. Library. osaka-u. ac. jp/

Osaka University 


\title{
Transport and Thermal Properties of Heavy-Fermion Superconductors: A Unified Picture
}

\author{
S. Schmitt-Rink, K. Miyake, ${ }^{(a)}$ and C. M. Varma \\ AT\& T Bell Laboratories, Murray Hill, New Jersey 07974 \\ (Received 11 March 1986)
}

\begin{abstract}
The transport and thermal properties of heavy-fermion superconductors are explained in terms of (i) an anisotropic order parameter with a line or lines of nodes and (ii) an effective mean free path which, except for the lowest temperatures, is approximately temperature independent and of a similar magnitude as in the normal state. Such a mean free path is shown to arise in a consistent treatment of impurity scattering close to the unitarity limit.
\end{abstract}

PACS numbers: $74.30 .-\mathrm{e}, 72.10 . \mathrm{Bg}, 74.20 . \mathrm{Fg}$

In the last few years, much attention has been focused on heavy-fermion compounds and their superconductivity. ${ }^{1-3}$ Arguments have been given ${ }^{4}$ that superconductivity cannot be due to conventional pairing, and that the pair wave function must have a zero at the origin of the relative coordinate. The pairing could then be in a triplet odd-parity state ${ }^{4}$ as in superfluid ${ }^{3} \mathrm{He}$ or in a singlet anisotropic state. ${ }^{5}$ Various experimental results on the thermodynamic and transport properties of $\mathrm{UPt}_{3}, \mathrm{UBe}_{13}$, and $\mathrm{CeCu}_{2} \mathrm{Si}_{2}$ bear out these suggestions. The particular superconducting state adopted is, however, not clear. In this paper we show that there is a consistent explanation of all the experimental observations in terms of a superconducting order parameter which has a line or lines of nodes (a polarlike state) on the Fermi surface and an $e f$ fective mean free path which is approximately temperature independent and almost the same as in the normal state over most of the range of the measurements. States with a gap all around the Fermi surface or with point nodes (axial-like states) do not consistently explain the observations, and the requirement on the effective mean free path is contrary to the results of impurity scattering in the Born approximation. ${ }^{6}$ We adopt the suggestion of Pethick and Pines ${ }^{7}$ and consider impurity scattering in the unitarity limit. We believe that this is an intrinsic feature of impurities in a Kondo lattice, where each magnetic ion leads at low temperatures to a conduction-electron phase shift of $\pi / 2$, the net effect being zero because of periodicity. A non-Kondo ion in such a lattice would then appear to offer a phase shift $\pi / 2$ with respect to the background.

In superconductors, the scattering of electrons from impurities is usually described by the Abrikosov-Gorkov (AG) theory. ${ }^{8}$ In the case of pair-breaking impurities a better calculation includes the effect of superconducting correlations on the scattering amplitude. This is well known from the theory of magnetic impurities in ordinary superconductors ${ }^{9}$ and the theory of nonmagnetic impurities in the Balian-Werthamer phase (of ${ }^{3} \mathrm{He}$ ). ${ }^{10}$ In these examples, the multiple scattering of electrons from impurities leads to the formation of quasiparticle bound states, which at finite impurity concentrations form impurity bands. In anisotropic superconductors with a finite quasiparticle density of states at all energies, the formation of quasiparticle bound states is not possible. However, for large coupling constants a scattering resonance may develop at low energies. This influences the transport properties in a decisive way.

To leading order in the impurity concentration $c$, the particle-hole-symmetric part of the electron self-energy is given by,10 $\Sigma(z)=c[T(z)-T(-z)] / 2$, where $T(z)=V+V \sum_{\mathbf{k}} G(\mathbf{k}, z) T(z)$ is the single-impurity $t$ matrix. Here, we assumed a contact impurity potential $V$ and odd-parity pairing. (No essential difference arises for anisotropic even-parity pairing.) $G$ is the normal part of the electron Green's function and $z$ the Matsubara frequency. Following AG, the effects of a finite concentration of impurities are treated by our making these equations self-consistent by renormalization of the Matsubara frequency according to $\tilde{z}=z-\Sigma(\tilde{z})$. The density of states (DOS) is given by

$$
N(E) / N_{\mathrm{F}}=-\left.\pi^{-1} \operatorname{Im} \sum_{\mathbf{k}} G(\mathbf{k}, \tilde{z})\right|_{z=E+i 0}
$$

and the transport coefficients can be calculated following Kadanoff and Falko ${ }^{11}$ and Ambegaokar and Griffin. ${ }^{12}$ For example, the average thermal conductivity $K$ $=\operatorname{tr} \hat{K} / 3$ is given by

$$
\frac{K}{K_{N}}=\frac{3}{2 \pi^{2} T^{3}} \int_{0}^{\infty} d E E^{2} \operatorname{sech}^{2}\left(\frac{E}{2 T}\right) L(E),
$$

where (neglecting vertex corrections)

$$
L(E)=\int \frac{d \Omega}{4 \pi} \frac{1}{2}\left(1+\frac{|\tilde{E}|^{2}-|\Delta(\Omega)|^{2}}{\left.\left|\tilde{E}^{2}-\right| \Delta(\Omega)\right|^{2} \mid}\right) \frac{1}{\operatorname{Im} 2 \tau_{N}\left[\tilde{E}^{2}-|\Delta(\Omega)|^{2}\right]^{1 / 2}} .
$$

In the limit $c \rightarrow 0,(1)$ and (3) can be identified with $\langle d \varepsilon / d E\rangle$ and $\left\langle(d E / d \varepsilon) \tau(E) / \tau_{N}\right\rangle$, respectively, where angular brackets denote the angular average over the region $|\Delta(\Omega)| \leq E, E=\left[\varepsilon^{2}+|\Delta(\Omega)|^{2}\right]^{1 / 2}, \varepsilon$ being the electron kinetic energy measured from the Fermi level, and $\tau(E)$ is the quasiparticle scattering time. For normal Fermi liquids $L(E)$ is 
the normalized energy-dependent mean free path.

We have determined $\tilde{E}$ and $\Delta$ self-consistently and evaluated $N(E) / N_{\mathrm{F}}, L(E)$, and the transport coefficients for the polar and the axial states. Figure 1(a) shows the results for the unperturbed and perturbed $N(E) / N_{\mathrm{F}}$ and $L(E)$ in the unitarity limit, $V N_{\mathrm{F}} \gg 1$, for a value $10^{-2}$ of the pair-breaking parameter $\eta=\left(2 \tau_{N} \Delta\right)^{-1}$ and a polar state. For the small but realistic $\eta$ chosen, $N(E) / N_{\mathrm{F}}$ exhibits a peak at the scattering resonance which in the unitarity limit is situated at $E=0$. Otherwise, it is almost identical to its unperturbed value and thus linear up to $E \approx \Delta$. The remarkable result is that $L(E)$ shows almost the same behavior as (the unperturbed) $N(E) / N_{\mathrm{F}}$, except at the lowest energies $E<10^{-1} \Delta$, where it saturates. Above this value, $L(E)$ may be fitted well by a straight line. Within standard kinetic theory one would identify $L(E)$ with the product of the quasiparticle density of states and the effective mean free path $l$ or relaxation time $\tau$, normalized to its normal state value. If we divide $L(E)$ by $N(E) / N_{\mathrm{F}}$ we find that $l / l_{N}$ or $\tau / \tau_{N}$ decreases with decreasing energy, the change be-

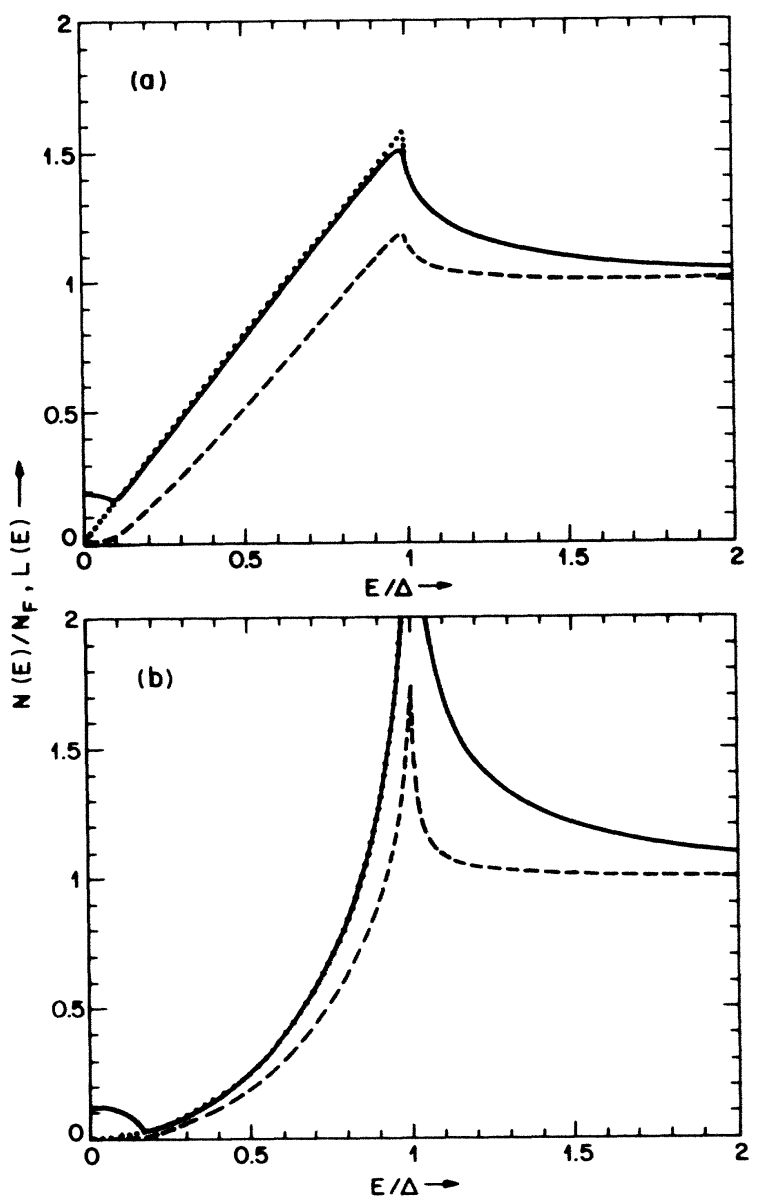

FIG. 1. Unperturbed (dotted curves) and perturbed (solid curves) density of states $N(E) / N_{\mathrm{F}}$, and $L(E)$ (dashed curves), in the unitarity limit for the (a) polar and (b) axial states and $2 \tau_{N} \Delta=10^{2}$. ing small (about a factor of 2) down to the pair-breaking region, $E<10^{-1} \Delta$. Much the same can be said for the axial state [Fig. 1(b)], and this behavior persists for all $\eta \ll 1$.

Pethick and Pines ${ }^{7}$ calculated the $t$ matrix in the unitarity limit, but with an unrenormalized $G(\mathbf{k}, z)$. Consequently, for the small impurity concentrations under consideration, their results should differ from ours mainly at low energies, where the effects of pair breaking are strongly enhanced. As for the transport properties, they draw their conclusions from the low-energy behavior only and find $\tau \sim T \ln ^{2}(\Delta / T)$ and $\tau \sim$ const for the polar and axial states, respectively. They conclude on the basis of such $\tau(T)$ that no form of superconducting order parameter explains the experiments consistently. On the other hand, we find for both states that at low energies $\tau \sim N(0)$, the impurity-induced DOS, and $\tau \sim$ constant and nearly equal to $\tau_{N}$ in the energy region above the pair-breaking regime relevant to most of the existing experiments.

Now we will discuss briefly the existing experimental data and show its consistency with the present theory assuming a polarlike state. A detailed comparison of our theory (including vertex corrections) and the experimental data will be published elsewhere.

Thermal conductivity, K.-The thermal conductivity $K$ has been measured in polycrystalline $\mathrm{UPt}_{3},{ }^{13,14}$ $\mathrm{UBe}_{13},{ }^{15}$ and $\mathrm{CeCu}_{2} \mathrm{Si}_{2}{ }^{16}$ samples and has been fitted by a $T^{2}$ behavior down to temperatures as low as $T_{c} / 10$. Figure 2 shows $K / K_{N}$ for the polar state and $\tau_{c} / \tau_{N}=2$ $\times 10^{-2}$, where $K_{N} \sim T$ and $\tau_{c}$ is the critical scattering time for the vanishing of superconductivity. As expected

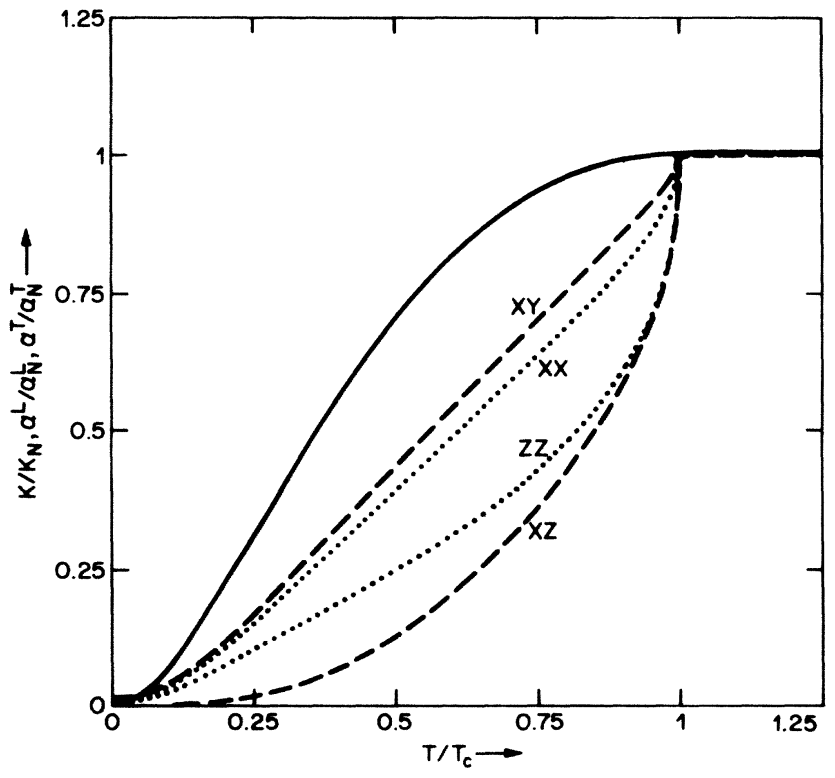

FIG. 2. Thermal conductivity $K / K_{N}$ (solid curve), longitudinal sound attenuation $\alpha^{L} / \alpha K$ (dotted curves), and transverse sound attenuation $\alpha^{T} / \alpha T$ (dashed curves) in the unitarity limit for the polar state and $\tau_{c} / \tau_{N}=2 \times 10^{-2}$. 
from the behavior of $L(E), K / K_{N}=O\left(T / T_{c}\right)$ over a large temperature range, which is in good agreement with the experimental results. The axial state in the same range gives $K / K_{N}=O\left(T^{2} / T_{c}^{2}\right){ }^{7}$ Recent experiments ${ }^{17}$ show a crossover to a $T$-independent $K / K_{N}$ in $\mathrm{UBe}_{13}$ at $T / T_{c} \sim 10^{-1}$, as predicted here.

Transverse ultrasonic attenuation, $\alpha^{T}$. Experiments have been performed in the hydrodynamic limit on $\mathrm{UPt}_{3}$ single crystals. ${ }^{18,19}$ Transverse sound has been propagated both along the $c$ axis ${ }^{18}$ and in the basal plane. ${ }^{19}$ In the first case the attenuation down to $T_{c} / 2$ has been found to vary like $T^{3}$, whereas in the second case both a $T$ (polarization in the basal plane) and a $T^{3}$ (polarization along the $c$ axis) power law have been deduced. Figure 2 shows $\alpha_{x y}^{T} / \alpha_{N}^{T}$ and $\alpha_{x z}^{T} / \alpha_{N}^{T}$ for the polar state and $\tau_{c} / \tau_{N}=2 \times 10^{-2}$, where $\alpha_{N}^{T} \sim$ constant. Here, $z$ and $x, y$ refer to the directions parallel and perpendicular to the polar axis, respectively. As expected, $\alpha_{x y}^{T} / \alpha_{N}^{T}$ $=O\left(T / T_{c}\right)$ and $\alpha_{x z}^{T} / \alpha_{N}^{T}=O\left(T^{3} / T_{c}^{3}\right)$ over a large temperature range, which is again in good agreement with the experiment. We can deduce that the line or lines of zeros of the gap are perpendicular to the $c$ axis.

Longitudinal ultrasonic attenuation, $\alpha^{L}$.-The first longitudinal ultrasonic attenuation measurements ${ }^{20}$ along the $c$ axis in $\mathrm{UPt}_{3}$ could be fitted well by a $T^{2}$ law between $T_{c} / 2$ and $T_{c} / 8$, which was the lowest temperature achieved. In the same temperature region, a better quality crystal, as judged from the width of the transition, yields a power law closer to $T^{1.5} .^{21}$ An even better quality crystal shows a pronounced peak just below $T_{c}$ followed by a $T^{3}$ dependence down to $T_{c} / 2 .^{18}$ The peak is also observed in $\mathrm{UBe}_{13},{ }^{22}$ with a similar power-law behavior at low temperatures. Figure 2 shows $\alpha_{x x}^{L} / \alpha_{N}^{L}$ and $\alpha_{z z}^{L} / \alpha_{N}^{L}$ for the polar state and $\tau_{c} / \tau_{N}=2 \times 10^{-2}$, where $\alpha_{N}^{L} \sim$ constant. As expected, $\alpha_{x x}^{L} / \alpha_{N}^{L}=O\left(T / T_{c}\right)$ and $\alpha_{z z}^{L} / \alpha_{N}^{L}=O\left(T / T_{c}\right)$ over a large temperature range. [For the axial state one finds $O\left(T^{2} / T_{c}^{2}\right){ }^{7,23}$ ] To explain the experimental results completely, the contribution near and below $T_{c}$ due to the relaxational mode of the order-parameter amplitude (Landau-Khalatnikov mechanism) must be included. ${ }^{24}$ This explains the peak observed just below $T_{c}$, but it also gives a low-temperature tail in $\alpha^{L} \sim T^{3}$ (again, with an effective constant $l$ or $\tau$ ).

A polarlike state has been invoked to explain the NMR experiments in $\mathrm{CeCu}_{2} \mathrm{Si}_{2}{ }^{25}$ and $\mathrm{UBe}_{13}{ }^{26}$ which show that $T_{1}^{-1} \sim T^{3}$ (for axial-like states, $T_{1}^{-1} \sim T^{5}$ ). The experimental results on the specific heat $C$ in $\mathrm{UBe}_{13}{ }^{27}$ and $\mathrm{CeCu}_{2} \mathrm{Si}_{2}{ }^{28}$ cannot be fitted by simple integral power laws, whereas in $\mathrm{UPt}_{3} C \sim T^{2}$ is found, again characteristic of a polarlike state. ${ }^{13,14}$ The behavior in $\mathrm{UBe}_{13}$ and $\mathrm{CeCu}_{2} \mathrm{Si}_{2}$ might be explained by the fact that the renormalizations ${ }^{29}$ have not attained their asymptotic values in the range of the experiments.

In conclusion, we have discussed how the assumption of a polarlike state, together with a consistent treatment of impurity scattering in the unitarity limit, leads to re- sults that are consistent with the experimental observations in all three compounds studied. We have presented calculations with a polar state as representative of the class of states with a line or lines of zeros of the gap. We cannot distinguish a specific polar-type state nor draw any conclusions from the experimental data about whether the pairing is in a triplet or an (anisotropic) singlet state. ${ }^{30}$ It is remarkable that three materials with different crystal structures, effective masses, spin orbitcoupling parameters, etc., should have similar anisotropic superconducting states. It is noteworthy that anisotropic superconductivity through exchange of antiferromagnetic spin fluctuations promotes even-parity states, all of which have line nodes of the gap. ${ }^{31}$

We have benefitted from numerous discussions with B. Batlogg, D. J. Bishop, B. Golding, and A. E. Ruckenstein. P. Hirschfeld, D. Vollhardt, and P. Wölfle have independently arrived at conclusions parallel to ours.

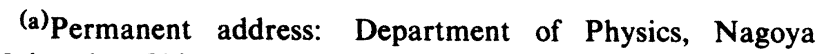
University, Chikusa-Ku, Nagoya 464, Japan.

${ }^{1}$ G. R. Stewart, Rev. Mod. Phys. 56, 755 (1984).

${ }^{2} \mathrm{~F}$. Steglich, in Theory of Heavy Fermions and Valence Fluctuations, Springer Series in Solid State Sciences Vol. 62, edited by M. Cardona, P. Fulde, H. J. Queisser, and K. von Klitzing (Springer, New York, 1985), p. 23.

${ }^{3}$ C. M. Varma, Comments Solid State Phys. 11, 221 (1985).

${ }^{4}$ C. M. Varma, Bull. Am. Phys. Soc. 29, 404 (1984); P. W. Anderson, Phys. Rev. B 30, 1549, 4000 (1984).

${ }^{5}$ K. Miyake, T. Matsuura, H. Jichu, and Y. Nagaoka, Prog. Theor. Phys. 72, 1063 (1984); T. Matsuura, K. Miyake, H. Jichu, Y. Kuroda, and Y. Nagaoka, J. Magn. Magn. Mater. 52, 239 (1985); F. J. Ohkawa and H. Fukuyama, J. Phys. Soc. Jpn. 53, 4344 (1984).

${ }^{6}$ L. Coffey, T. M. Rice, and K. Ueda, J. Phys. C 18, L813 (1985).

${ }^{7}$ C. J. Pethick and D. Pines, Phys. Rev. Lett. 57, 118 (1986).

${ }^{8}$ A. A. Abrikosov and L. P. Gorkov, Zh. Eksp. Teor. Fiz. 39, 1781 (1960) [Sov. Phys. JETP 12, 1243 (1961)].

${ }^{9}$ H. Shiba, Prog. Theor. Phys. 40, 435 (1968); J. Zittartz, A. Bringer, and E. Müller-Hartmann, Solid State Commun. 10, 513 (1972).

${ }^{10}$ L. J. Buchholtz and G. Zwicknagl, Phys. Rev. B 23, 5788 (1981); E. V. Thuneberg, J. Kurkijärvi, and D. Rainer, Physica B (Amsterdam) 107, 43 (1981).

${ }^{11}$ L. P. Kadanoff and I. I. Falko, Phys. Rev. 136, A1170 (1964).

${ }^{12}$ V. Ambegaokar and A. Griffin, Phys. Rev. 137, A1151 (1965).

${ }^{13}$ D. Jaccard, J. Flouquet, P. Lejay, and J. L. Tholence, J. Appl. Phys. 57, 3082 (1985).

${ }^{14}$ J. J. M. Franse, A. Menovsky, A. de Visser, C. D. Bredl, U. Gottwick, W. Lieke, H. M. Mayer, U. Rauchschwalbe, G. Sparn, and F. Steglich, Z. Phys. B 59, 15 (1985).

${ }^{15}$ D. Jaccard and J. Flouqet, J. Magn. Magn. Mater 47/48, 45 (1985).

${ }^{16}$ G. Sparn, W. Lieke, U. Gottwick, F. Steglich, and N. Grewe, J. Magn. Magn. Mater. 47/48, 521 (1985). 
${ }^{17} \mathrm{D}$. Jaccard and J. Flouquet, to be published.

${ }^{18}$ V. Müller, D. Maurer, E. W. Scheidt, Ch. Roth, K. Lüders, E. Bucher, and H. E. Bömmel, Solid State Commun. 57, 319 (1986).

${ }^{19}$ B. S. Shivaram, Y. H. Jeong, T. F. Rosenbaum, and D. J. Hinks, Phys. Rev. Lett. 56, 1078 (1986).

${ }^{20}$ D. J. Bishop, C. M. Varma, B. Batlogg, E. Bucher, Z. Fisk, and J. L. Smith, Phys. Rev. Lett. 53, 1009 (1984).

${ }^{21}$ D. J. Bishop, private communication.

${ }^{22}$ B. Golding, D. J. Bishop, B. Batlogg, W. H. Haemmerle, Z. Fisk, J. L. Smith, and H. R. Ott, Bull. Am. Phys. Soc. 30, 1357 (1985), and Phys. Rev. Lett. 55, 2479 (1985).

${ }^{23}$ J. P. Rodriguez, Phys. Rev. Lett. 55, 250 (1985).

${ }^{24}$ K. Miyake and C. M. Varma, Phys. Rev. Lett. (to be published).

${ }^{25}$ Y. Kitaoka, K. Ueda, T. Kohara, K. Asayama, Y. Onuki, and T. Komatsubara, J. Magn. Magn. Mater. 52, 341 (1985).

${ }^{26}$ D. E. MacLaughlin, C. Tien, W. C. Clark, M. D. Lan, Z. Fisk, J. L. Smith, and H. R. Ott, Phys. Rev. Lett. 53, 1833 (1984).

${ }^{27}$ H. R. Ott, H. Rudigier, T. M. Rice, K. Ueda, Z. Fisk, and J. L. Smith, Phys. Rev. Lett. 52, 1915 (1984).

${ }^{28}$ F. Steglich, C. D. Bredl, W. Lieke, U. Rauschschwalbe, and G. Sparn, Physica B (Amsterdam) 126, 82 (1984).

${ }^{29}$ C. M. Varma, K. Miyake, and S. Schmitt-Rink, Phys. Rev. Lett. 57, 626 (1986).

30Polarlike states in the triplet manifold appear not to be allowed. See, e.g., E. I. Blount, Phys. Rev. B 32, 2935 (1985); G. E. Volovik and L. P. Gorkov, Pis'ma Zh. Eksp. Teor. Fiz. 39, 550 (1984) [JETP Lett. 39, 674 (1984)].

${ }^{31}$ K. Miyake, S. Schmitt-Rink, and C. M. Varma, Phys. Rev. B (to be published). 